\title{
Low expression of CHRDL1 and SPARCL1 predicts poor prognosis of lung adenocarcinoma based on comprehensive analysis and immunohistochemical validation
}

Huan Deng ${ }^{1,2,3,4 \dagger}$, Qingqing Hang ${ }^{2,3,4,5+}$, Dijian Shen ${ }^{2,3,4}$, Yibi Zhang ${ }^{6}$ and Ming Chen ${ }^{1,2,3,4^{*}}$ (D)

\begin{abstract}
Purpose: Exploring the molecular mechanisms of lung adenocarcinoma (LUAD) is beneficial for developing new therapeutic strategies and predicting prognosis. This study was performed to select core genes related to LUAD and to analyze their prognostic value.
\end{abstract}

Methods: Microarray datasets from the GEO (GSE75037) and TCGA-LUAD datasets were analyzed to identify differentially coexpressed genes in LUAD using weighted gene coexpression network analysis (WGCNA) and differential gene expression analysis. Functional enrichment analysis was conducted, and a protein-protein interaction (PPI) network was established. Subsequently, hub genes were identified using the CytoHubba plug-in. Overall survival (OS) analyses of hub genes were performed. The Clinical Proteomic Tumor Analysis Consortium (CPTAC) and the Human Protein Atlas (THPA) databases were used to validate our findings. Gene set enrichment analysis (GSEA) of survival-related hub genes were conducted. Immunohistochemistry (IHC) was carried out to validate our findings.

Results: We identified 486 differentially coexpressed genes. Functional enrichment analysis suggested these genes were primarily enriched in the regulation of epithelial cell proliferation, collagen-containing extracellular matrix, transforming growth factor beta binding, and signaling pathways regulating the pluripotency of stem cells. Ten hub genes were detected using the maximal clique centrality (MCC) algorithm, and four genes were closely associated with OS. The CPTAC and THPA databases revealed that CHRDL1 and SPARCL1 were downregulated at the MRNA and protein expression levels in LUAD, whereas SPP1 was upregulated. GSEA demonstrated that DNA-dependent DNA replication and catalytic activity acting on RNA were correlated with CHRDL1 and SPARCL1 expression, respectively. The IHC results suggested that CHRDL1 and SPARCL1 were significantly downregulated in LUAD.

Conclusions: Our study revealed that survival-related hub genes closely correlated with the initiation and progression of LUAD. Furthermore, CHRDL1 and SPARCL1 are potential therapeutic and prognostic indicators of LUAD.

Keywords: Lung adenocarcinoma, Weighted gene coexpression network analysis, Differential coexpression genes, Protein-protein interaction network, Survival analysis

*Correspondence: chenming@zjcc.org.cn

†Huan Deng and Qingqing Hang are the co-first authors of this manuscript

${ }^{1}$ College of Life Sciences, University of the Chinese Academy of Sciences, Beijing 100049, China

Full list of author information is available at the end of the article

\section{Introduction}

As one of common cancers, lung carcinoma was estimated to have caused disease in 235,760 patients and 131,880 deaths in 2021, resulting in a tremendous burden to our society [1]. Patients with NSCLC

(c) The Author(s) 2021. This article is licensed under a Creative Commons Attribution 4.0 International License, which permits use, sharing, adaptation, distribution and reproduction in any medium or format, as long as you give appropriate credit to the original author(s) and the source, provide a link to the Creative Commons licence, and indicate if changes were made. The images or other third party material in this article are included in the article's Creative Commons licence, unless indicated otherwise in a credit line to the material. If material is not included in the article's Creative Commons licence and your intended use is not permitted by statutory regulation or exceeds the permitted use, you will need to obtain permission directly from the copyright holder. To view a copy of this licence, visit http://creativeco mmons.org/licenses/by/4.0/. The Creative Commons Public Domain Dedication waiver (http://creativecommons.org/publicdomain/ zero/1.0/) applies to the data made available in this article, unless otherwise stated in a credit line to the data. 
accounted for nearly $85 \%$ of all patients with lung cancer, and the most prevalent pathological pattern of NSCLC was lung adenocarcinoma (LUAD) [2]. In recent decades, many researchers have concentrated on studying the potential biological and molecular mechanisms of lung cancer, and the molecular mechanisms are gradually being understood [3]. It was recognized that identifying key molecular abnormalities would promote the rapid development of precision medicine, and more effective strategies will be identified for the diagnosis, treatment and prognosis of LUAD in the near future [4]. Currently, it is essential for us to identify core genes associated with the carcinogenesis and development of LUAD.

With the rapid advancement of genomic technology, bioinformatics analyses have been widely used in the analysis of microarray datasets to further study the potential molecular mechanisms of cancers and to identify tumor-specific indicators [5]. Weighed gene coexpression network analysis (WGCNA) is one of these significant algorithms that provides a better understanding of gene coexpression networks and gene functions [6]. WGCNA can detect modules of highly correlated genes among samples to relate modules to external sample traits, providing valuable insights into predicting possible functions of coexpressed genes [7]. Moreover, differential gene expression analysis is usually used in transcriptomics datasets to study underlying biological and molecular mechanisms and to identify quantitative differences in the expression level of the gene between different groups [8].

To improve the discriminating ability of highly related genes, the two methods mentioned above were applied in our study. First, mRNA expression datasets of LUAD were obtained from Gene Expression Omnibus (GEO) and The Cancer Genome Atlas database (TCGA). Second, WGCNA and differential gene expression analysis were used to identify common differential coexpression genes. Then, we carried out functional enrichment analysis, protein-protein interaction (PPI) analysis, and overall survival analysis to identify potential biomarkers correlated with the occurrence and progression of LUAD. Next, the expression patterns of hub genes at the mRNA and protein levels were verified through GSE19188 from GEO, Clinical Proteomic Tumor Analysis Consortium (CPTAC) and the Human Protein Atlas (HPA). Furthermore, we conducted gene set enrichment analyses (GSEA) of survival-related hub genes using the TCGA-LUAD dataset. Ultimately, we carried out immunohistochemistry (IHC) analysis of survivalrelated hub genes for further validation.

\section{Materials}

Figure 1 reveals the detailed processes of data download, hub gene identification and external validation. Every step is illustrated in the following subsection.

\section{Datasets from GEO and TCGA}

The mRNA expression datasets of LUAD were acquired from the GEO and TCGA databases. First, one microarray dataset (GSE75037) was selected from GEO, and this dataset included 83 LUAD tissues and 83 matched nonmalignant adjacent tissues from LUAD patients. GSE75037 was based on the GPL6884 Illumina HumanWG-6 v3.0 expression beadchip. Based on the manufacturer-provided annotation file, probes would be transformed to corresponding gene symbol, probe sets without gene symbol would be removed, and duplicated probes for the same gene would be averaged. Consequently, a total of 25,428 genes were acquired for the next analysis. Second, the mRNA expression data and corresponding clinical information of LUAD were acquired from TCGA. 594 LUAD samples were downloaded, consisting of 535 LUAD and 59 normal lung tissues, and RNAseq data about fragments per kilobase per million (FPKM) on 19,145 genes were obtained. Then, FPKM data were transformed to transcript per million (TPM) data for the next analysis. Based on the Illumina HiSeq 2000 platform, all the data were generated and annotated to a reference transcript set of Human hg38 gene standard tracks. The edgeR package tutorial showed that genes with low read counts were probably meaningless for the next analysis [9]. Therefore, genes with TPM $<1$ were removed from our study. As a result, 15,142 genes were obtained for the subsequent analysis.

\section{Identification of core coexpression modules through WGCNA}

We constructed gene coexpression networks of the GSE75037 and mRNA expression profiles of the TCGA-LUAD dataset through the WGCNA package. WGCNA was used to identify highly related genes and aggregate these genes into the same genetic module related to external sample traits. To construct a scale-free network, soft powers $\beta=14$ (Fig. $2 b$ ) and 6 (Fig. 3b) were used for GSE75037 and mRNA expression profiles of TCGA-LUAD, respectively. Then, the 


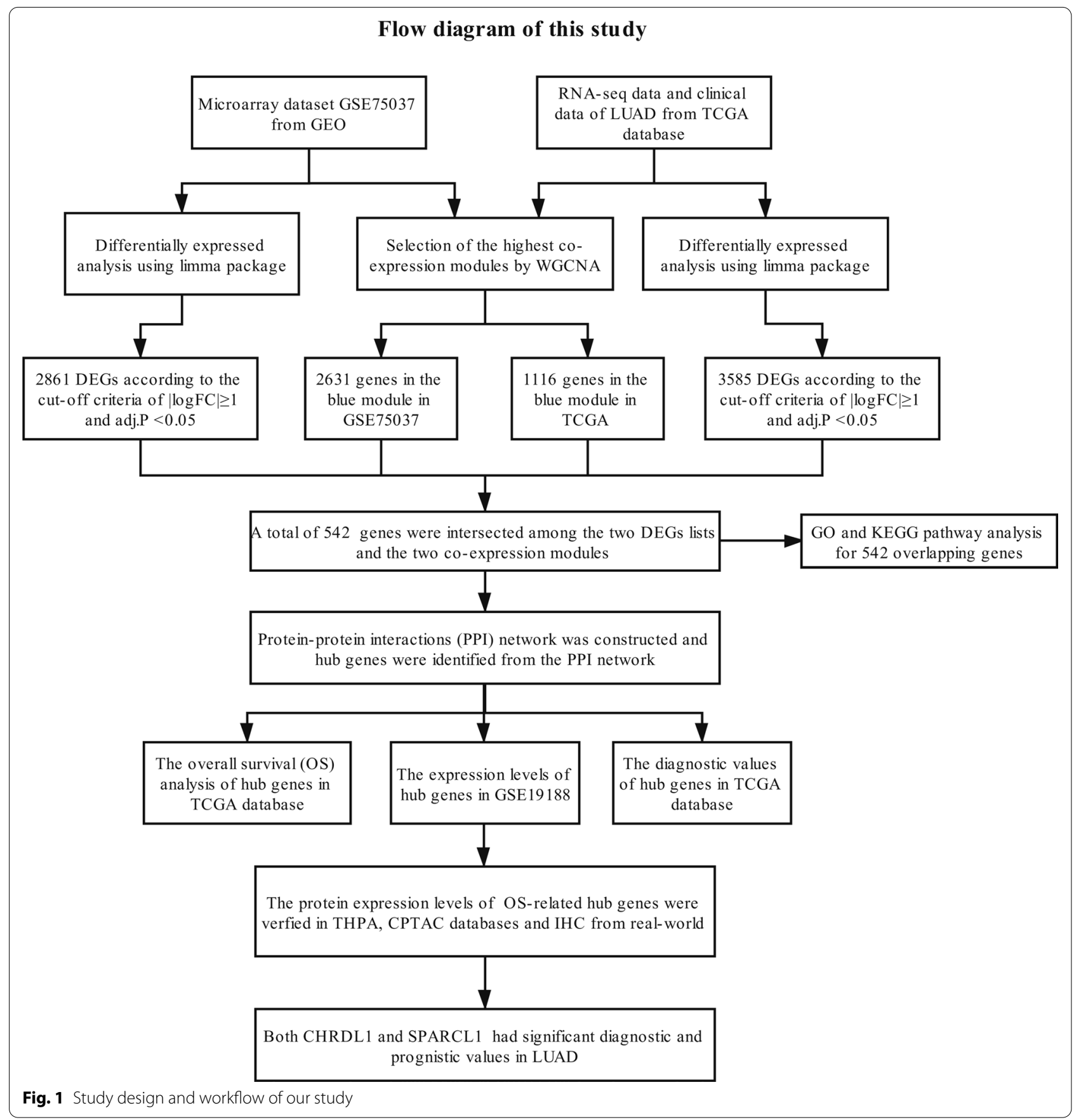

adjacency matrix was generated using the following formula: $a i j=|S i j|^{\beta}$ (aij: adjacency matrix between gene $i$ and gene $j$, Sij: similarity matrix, which is determined by Pearson correlation of all gene pairs, and $\beta$ : softpower value); then, the matrix was converted to a topological overlap matrix (TOM) and the corresponding dissimilarity (1-TOM). Subsequently, we established a hierarchical clustering dendrogram of the 1-TOM matrix to aggregate genes with similar expression into a coexpression module. The module-trait relations between modules and clinical trait information were explored for further identification 

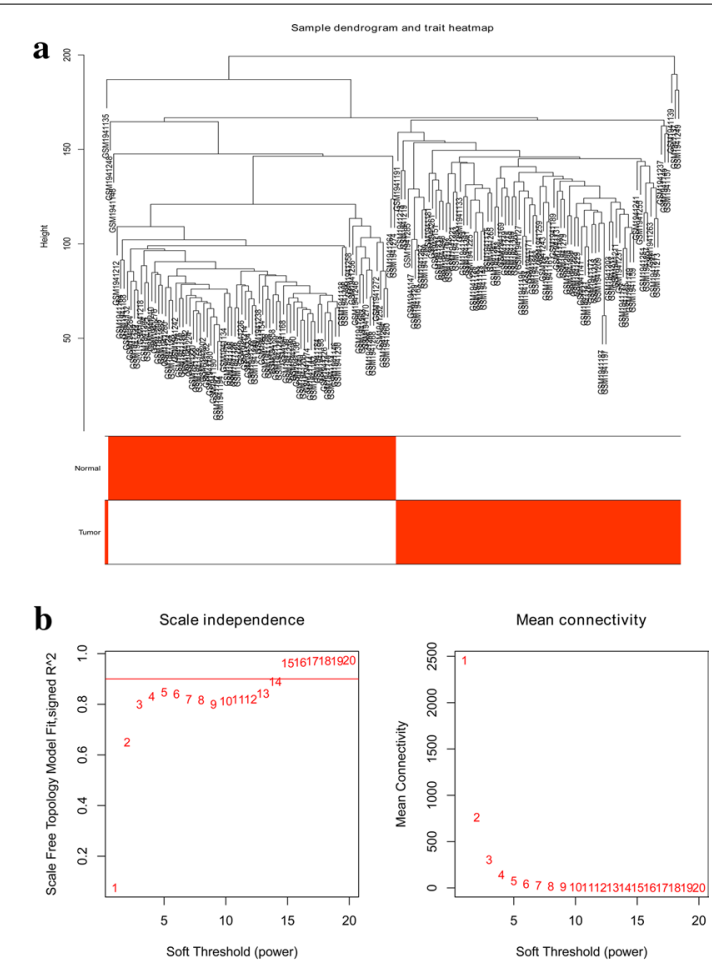

c

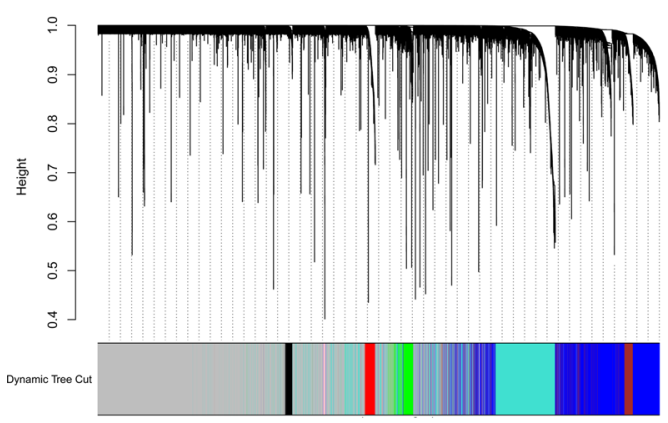

d

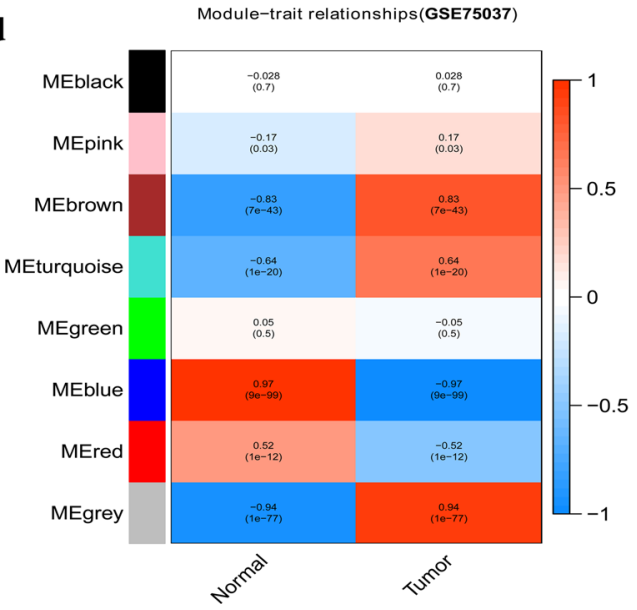

Fig. 2 Identification of modules correlated with the clinical traits in GSE75037. a Sample dendrogram and trait heatmap. b Scale independence and Mean connectivity. c The Cluster dendrogram of co-expression network modules is ordered by a hierarchical clustering of genes based on the 1-TOM matrix. Different colors represent different modules. $\mathbf{d}$ Module-trait relationships. Each row represents a color module and every column represents a clinical trait (normal and tumor). Each cell contains the corresponding correlation and P-value

of functional modules in the coexpression network. Thus, the module with the highest correlation coefficient was selected as the candidate module related to clinical traits, which was used for our next analysis.

\section{Selection of differential coexpression genes}

The limma and edgeR packages were applied to perform differential expression analysis of microarray and RNA-Sequencing datasets, respectively $[9,10]$. To select differentially expressed genes (DEGs) between LUAD tissues and nonmalignant tissues, we respectively used the limma and edge $R$ packages in the selection of DEGs from the GSE75037 and TCGA-LUAD datasets. To reduce the false discovery rate (FDR), the $\mathrm{p}$-value was adjusted using the Benjamini-Hochberg method. The selection criteria for DEGs were set as $|\log \mathrm{FC}|>1$ and adj. $\mathrm{P}<0.05$. To better discriminate highly related genes, we determined the intersection of genes among two lists of DEGs and two lists of coexpressed genes from the two coexpression networks, which were applied to identify candidate prognostic indicators of LUAD.

\section{Functional enrichment analysis}

To analyze the biological functions of differentially coexpressed genes, GO and KEGG pathway analysis was conducted with the clusterProfiler [11] and GOplot packages. GO and KEGG are essential bioinformatics tools, which annotates gene and analyzes the biological process of genes [12]. $\mathrm{P}<0.05$ was considered statistically significant.

\section{PPI network construction and hub gene selection}

The PPI network of differentially coexpressed genes was established with the Search Tool for the Retrieval of Interacting Genes (STRING) [13]. Cytoscape 

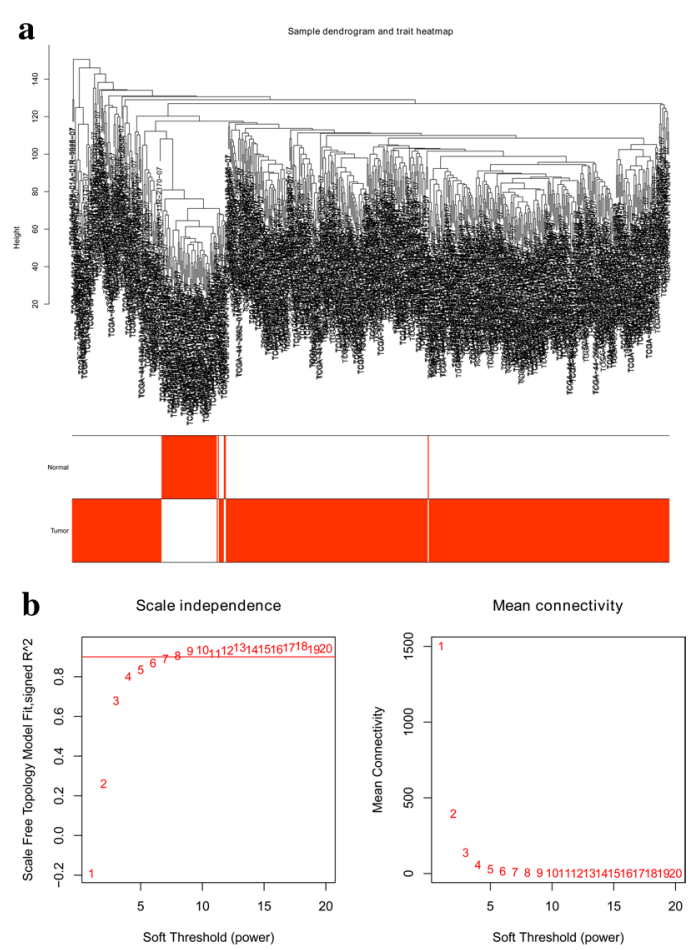

c

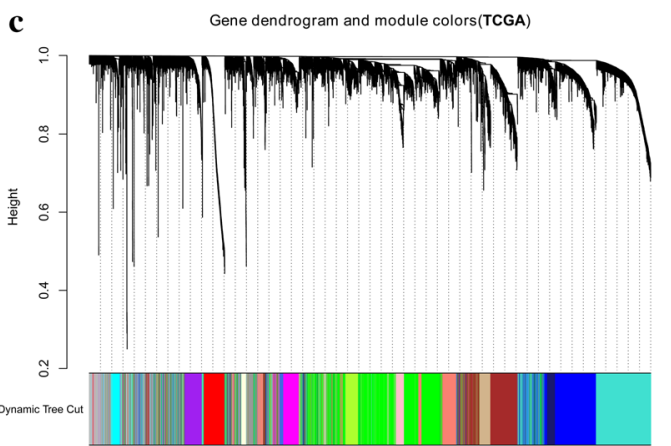

d

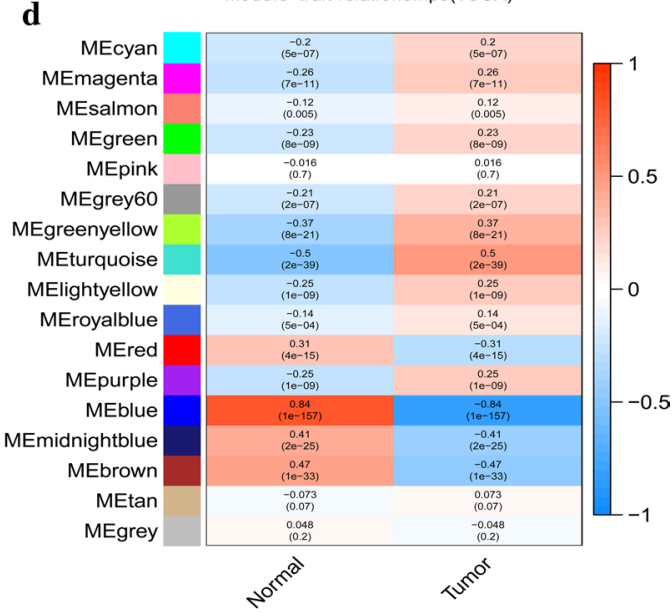

Fig. 3 Identification of modules correlated with the clinical traits in TCGA-LUAD dataset. a Sample dendrogram and trait heatmap. b Scale independence and mean connectivity. c The Cluster dendrogram of co-expression network modules is ordered by a hierarchical clustering of genes based on the 1-TOM matrix. Different colors represent different modules. d Module-trait relationships.

Each row represents a color module and every column represents a clinical trait (normal and tumor). Each cell contains the corresponding correlation and P-value

(version 3.7.2) was used to build a visual network of molecular interactions with a combined score $>0.6$ [14]. The Molecular Complex Detection (MCODE) plugin in Cytoscape was used to detect highly correlated modules in PPI network [15]. The most significant gene module was visualized and graphically displayed using the MCODE plug-in. The criteria of selection were as follows: MCODE score $>5$, node score cutoff $=0.2$, degree cutoff $=2$, $\mathrm{k}$-score $=2$, and $\max$ depth $=100$. Additionally, the maximal clique centrality (MCC) algorithm was recognized to be the most useful approach to detect hub nodes from the PPI network [16]. We calculated the MCC score of every gene in the PPI network using the CytoHubba plug-in. Differentially co-expressed genes with the top ten highest MCC scores were believed to be hub genes. These hub genes were also visualized using the CytoHubba plug-in.

\section{Prognostic roles and relationship with pathological stages of hub genes}

To explore the prognostic values of hub genes in LUAD, Kaplan-Meier univariate survival analysis was conducted through the survival package. Only patients with complete follow-up information were included for overall survival (OS) analysis, and we classified these patients into two cohorts in accordance with the median expression level of hub genes. Log-rank $\mathrm{p}<0.05$ was considered statistically significant. Additionally, we explored the relationship between their expression patterns and pathological stages among LUAD. 


\section{External validation of GEO, CPTAC and THPA databases}

To improve the reliability of our analysis, the GEO, CPTAC and THPA databases were used to validate the expression patterns of survival-related hub genes between LUAD and nonmalignant samples. To systematically analyze the mRNA expression patterns of survival-related hub genes between LUAD and nonmalignant samples, meta-analyses were carried out using relevant data from GEO. The search strategy and selection criteria for the included datasets in the GEO database are shown (Additional file 3: Table S1). Additionally, their protein expression patterns between LUAD and nonmalignant samples were explored using IHC outcomes from HPA [17] and quantitative comparison from CPTAC database [18].

\section{GSEA of survival-related hub genes}

We divided these samples into two cohorts according to the median expression values of hub genes associated with OS. The effect of the expression of hub genes on multiple gene sets was analyzed for related GO enrichment analysis using c5.all.v7.2.symbols. gmt [gene ontology] [19]. The permutation of each analysis was set to 1000 times. |Normalized enrichment score $(\mathrm{NES}) \mid>1$, NOM p-value $<0.05$ and FDR $\mathrm{q}$-value $<0.25$ were considered significant differences.

\section{Immunohistochemical verification}

Twenty pairs of LUAD and normal tissues had been collected in Zhejiang Cancer Hospital (Zhejiang, China) from 2017 to 2021 (Additional file 4: Table S2). IHC was approved by the Medical Ethics Committee of Zhejiang Cancer Hospital (IRB-2020-817). These tissue samples were frozen in liquid nitrogen for next analysis. After epitope retrieval, hydrogen peroxide treatment and nonspecific antigen blocking, we incubated the sections of these tissues via deparaffinization and dehydration using anti-CHRDL1 (dilution: 1:500, PA5-78591, Thermo, USA) and anti-SPARCL1 antibodies (dilution: 1:1000, ab255597, Abcam, UK) overnight at $4{ }^{\circ} \mathrm{C}$. Afterward, we incubated these sections using secondary antibodies (dilution: 1:200, ab150115, Abcam, UK). All sections were covered with Fluoroshield containing 4',6-diamidino-2-phenylindole (DAPI, Abcam) for $10 \mathrm{~min}$ to identify nuclei, and we detected the signal using the DAB staining kit (Vector Laboratories, USA). The intensity was denoted as 0 (negative), 1+ (weakly positive), $2+$ (moderately positive), and $3+$ (strongly positive). $\mathrm{H}$-score values (range $0-300$ ) were calculated according to the following formula: [(\% cells with an intensity of $1+)+2 \times(\%$ cells with an intensity of $2+)+3 \times(\%$ cells with an intensity of $3+$ )]. Two pathologists independently estimated scores of all sections, and mean scores were calculated as $\mathrm{H}$-score values. IHC was independently repeated in triplicate, and student's t-test was applied for comparisons between LUAD and normal lung tissue groups.

\section{Results}

Identification of core coexpression modules through WGCNA

To detect the functional modules in LUAD, we established two gene coexpression networks using the GSE75037 and TCGA-LUAD datasets through the WGCNA package in R software. We found eight modules (Fig. 2c) and 17 modules (Fig. 3c) in the GSE75037 and TCGA-LUAD datasets, respectively (one color represents one module). Next, the heatmaps explored the relationship between modules and two clinical traits (normal and LUAD) in the GSE75037 (Fig. 2d) and TCGA-LUAD datasets (Fig. 3d), suggesting that the blue module in GSE75037 and the blue module TCGA-LUAD dataset were closely associated with normal tissues (blue module in GSE75037: $r=0.97$, $\mathrm{p}=9 \mathrm{e}-99$; blue module in TCGA-LUAD dataset: $\mathrm{r}=0.84, \mathrm{p}=1 \mathrm{e}-157)$.

\section{The intersection of DEGs and coexpression genes}

The heatmaps showed the expression patterns of 50 upregulated and 50 downregulated genes in the GSE75037 (Fig. 4a) and TCGA-LUAD datasets (Fig. 4b). The volcano plots illustrated that 2861 DEGs in GSE75037 (Fig. 4c) and 3585 DEGs in TCGA-LUAD dataset (Fig. 4d) were significantly dysregulated.

(See figure on next page.)

Fig. 4 Identification of differentially expressed genes (DEGs) among GSE75037 TCGA-LUAD dataset with the cut-off criteria of $|\log F C|>1$ and adj.P $<0.05$. a Heatmap of 50 upregulated and 50 downregulated DEGs of GSE75037. b Heatmap of 50 upregulated and 50 downregulated DEGs of TCGA-LUAD dataset. c Volcano plot of DEGs in GSE75037. d Volcano plot of DEGs in TCGA-LUAD dataset. e The Venn diagram of genes among the two DEG lists and the two lists of co-expression genes. In total, 486 overlapping differential co-expression genes are found 

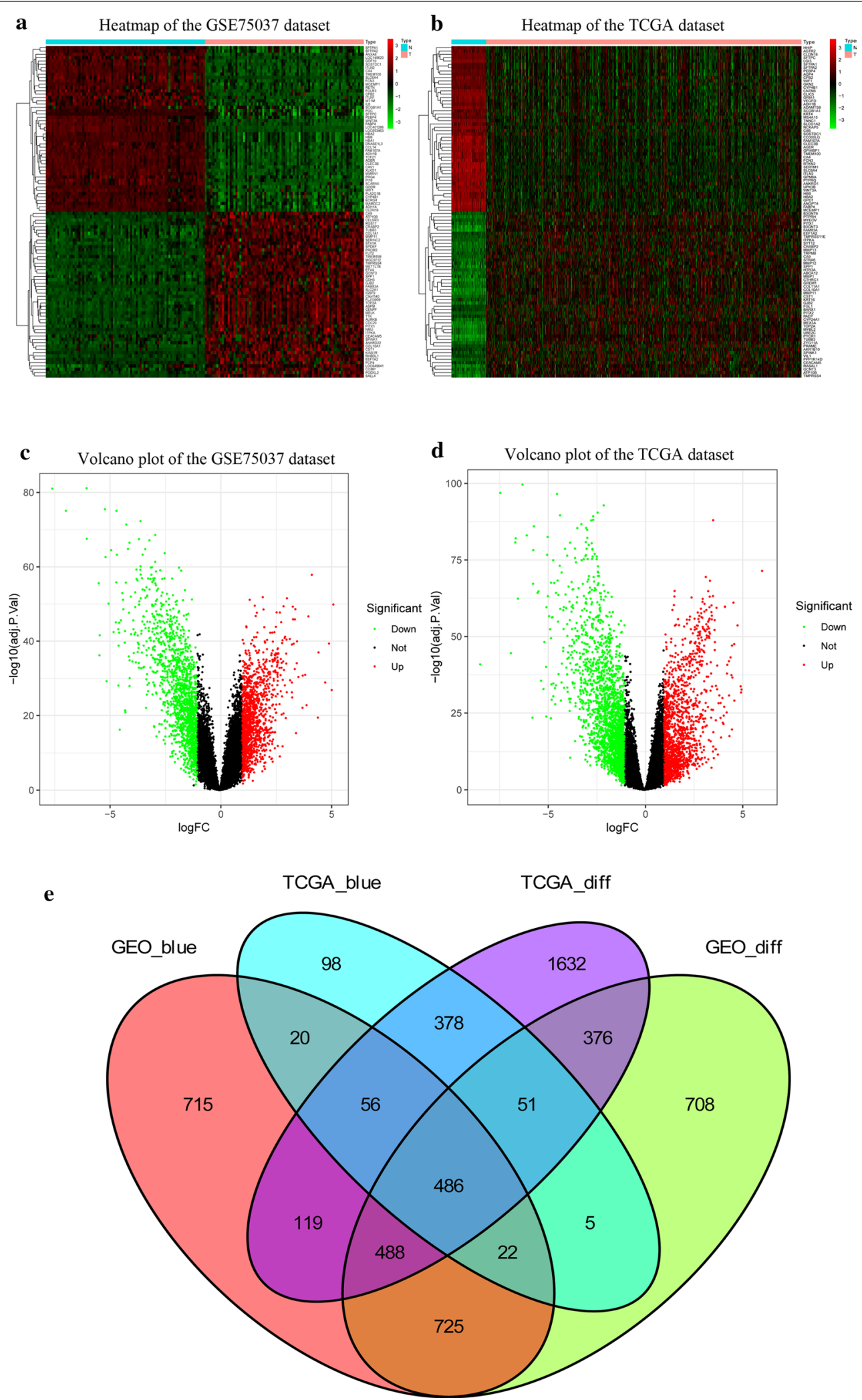


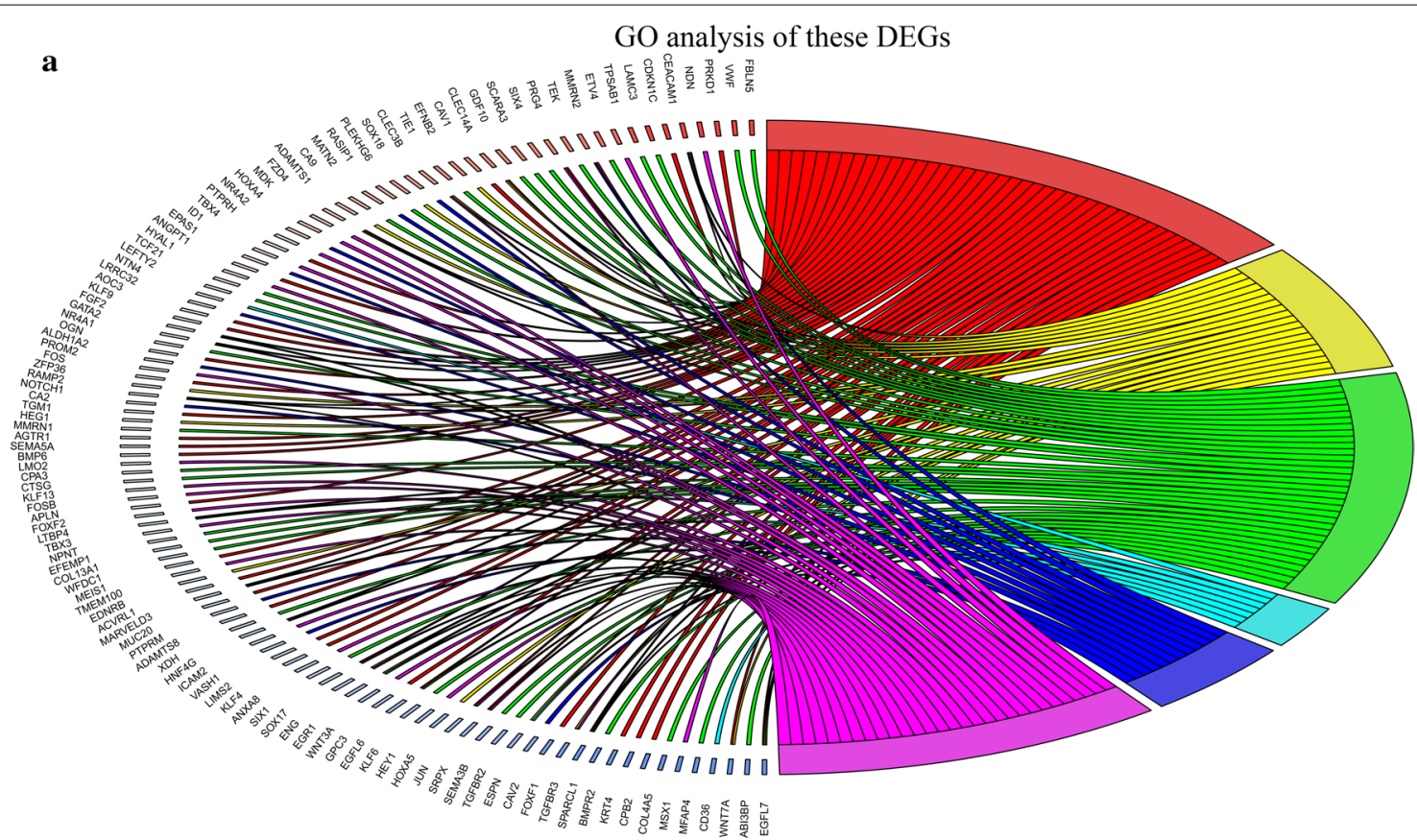

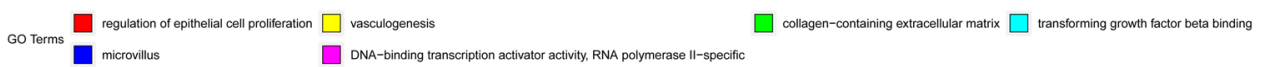

b

KEGG pathway of these DEGs

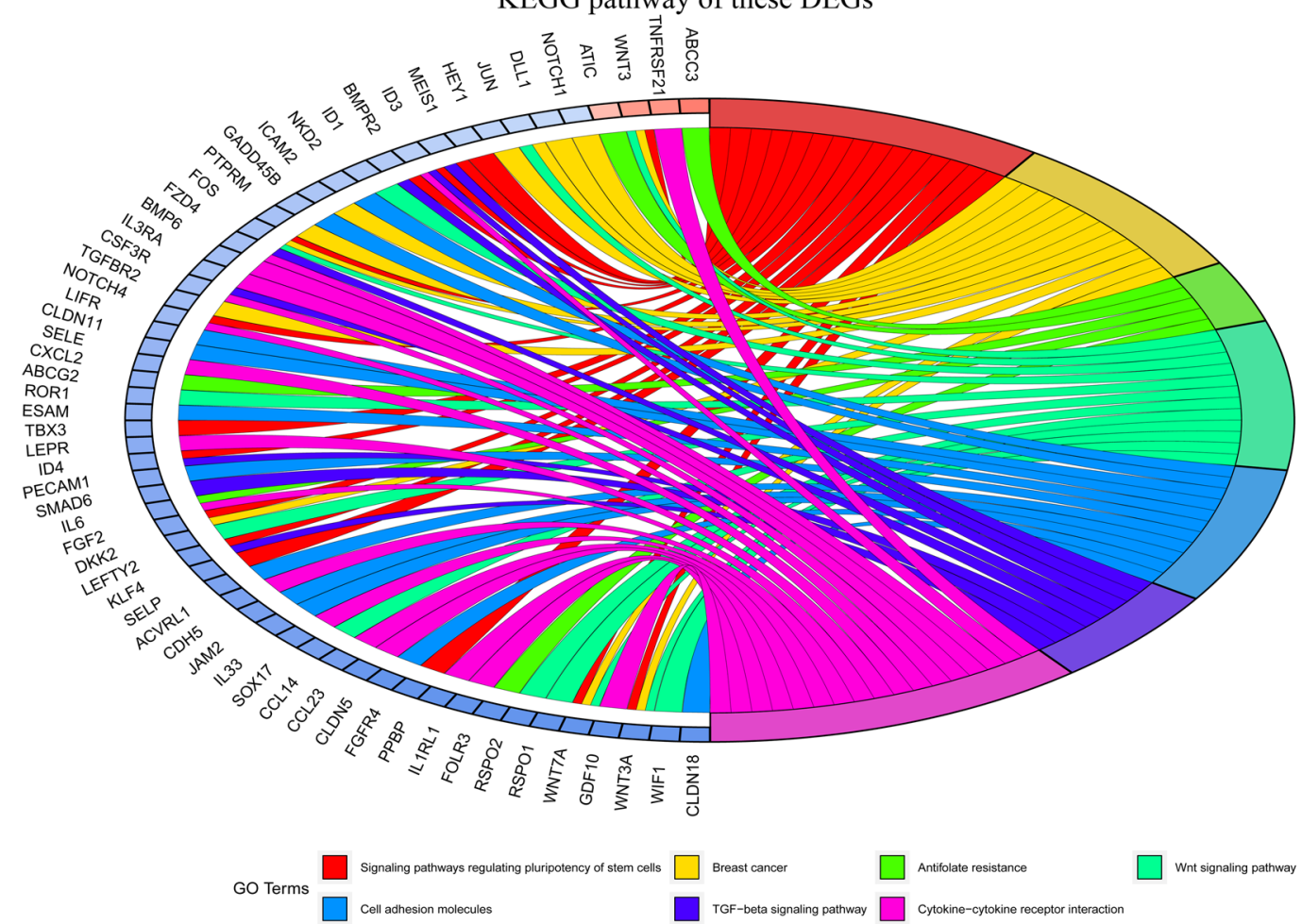

Fig. 5 Functional enrichment analysis of differential co-expression genes using the clusterProfiler package. a Gene ontology (GO) enrichment analysis of differential co-expression genes. b Kyoto encyclopedia of genes and genomes pathway (KEGG) of differential co-expression genes 


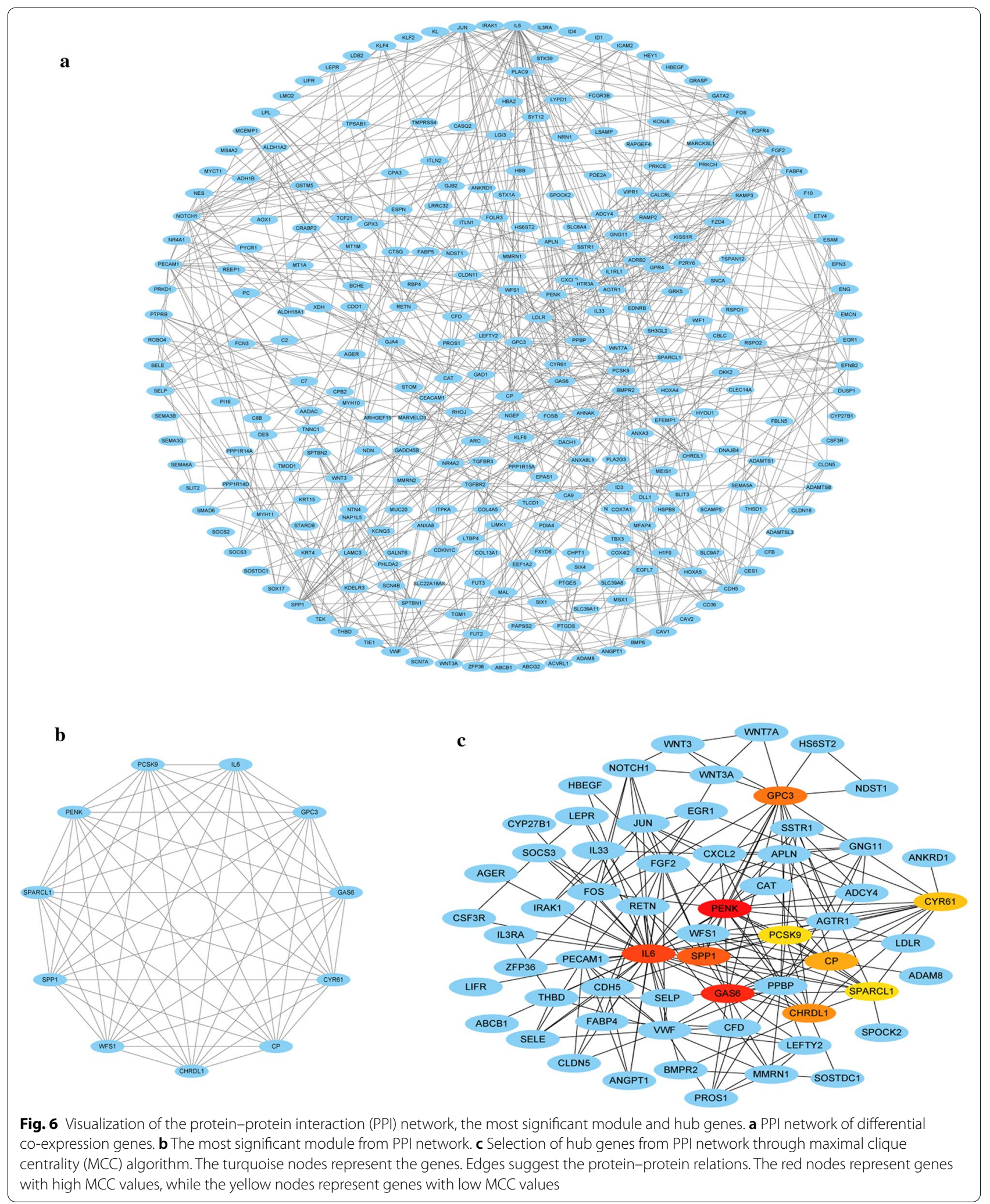


Figure 4e clearly demonstrates that the intersection of two lists of DEGs (Additional file 5: Table S3; Additional file 6: Table S4) and two lists of coexpressed genes (Additional file 7: Table S5; Additional file 8: Table S6) contained 486 genes, which were used for the subsequent analysis (Additional file 9: Table S7).

\section{Functional enrichment analysis of differentially coexpressed genes}

The outcomes of BP analysis of these genes showed that the regulation of epithelial cell proliferation and vasculogenesis were significantly enriched. CC analysis revealed that collagen-containing extracellular matrix and microvilli were associated with 486 genes. According to the outcomes of the MF analysis, transforming growth factor beta binding and DNA-binding transcription activator activity, RNA polymerase II-specific genes were primarily enriched (Fig. 5a). Furthermore, KEGG pathway results illustrated that signaling pathways regulating the pluripotency of stem cells, breast cancer and antifolate resistance were mainly enriched (Fig. 5b).

\section{PPI network construction and hub genes selection}

The PPI network of differentially coexpressed genes was displayed in Fig. 6a, which included 283 nodes and 632 edges. The most significant module was found using the MCODE plug-in, which contained 11 nodes and 55 edges (Fig. 6b). Then, hub genes were identified according to the rank of MCC values. The top 10 genes (PENK, GAS6, IL6, SPP1, GPC3, CHRDL1, CP, CYR61, WFS1 and SPARCL1) were recognized as hub genes. These hub genes selected from the PPI network are clearly illustrated in Fig. 6c, and the shade of the color represents the magnitude of the MCC scores.

\section{Prognostic roles of hub genes and relation with pathological stages}

To explore the prognostic roles of the top 10 hub genes in LUAD, survival analysis was performed using the survival information of the TCGA-LUAD dataset. Lower expression of CHRDL1, SPARCL1 and PENK correlated with the poor prognosis among LUAD patients, while higher expression of SPP1 was correlated with poor prognosis (Fig. 7a). In addition, we also explored the relationship between the expression levels of hub genes and pathological stages (Fig. 7b-e).

\section{External validation of public databases}

To increase the reliability of our findings, three external databases were used in our study. First, nine datasets satisfying the selection criteria were included for the comparisons of mRNA patterns of OS-related genes (Table 1). Comprehensive metaanalyses of the nine datasets indicated that CHRDL1 (Fig. 8a), SPARCL1 (Fig. 8b) and PENK (Fig. 8d) were downregulated in LUAD tissues, whereas SPP1 was upregulated (Fig. 8c). Second, we still compared the protein expression levels of survival-related genes in the CPTAC (Fig. 8e-g) and HPA (Additional file 1: Figure S1; Additional file 2: Figure S2) databases. Table 2 shows the detailed results of the IHC analysis of these genes based on the HPA database. Although the expression level of PENK was missing in the CPTAC database, the protein expression patterns of CHRDL1, SPARCL1, and SPP1 were consistent with their mRNA expression patterns.

\section{GSEA of survival-related hub genes}

GSEA showed that DNA-dependent DNA replication, mitotic metaphase plate congression, and mitotic sister chromatid segregation were associated with CHRDL1 (Fig. 9a). In addition, GSEA suggested that catalytic activity acting on RNA, DNA packing and mesenchymal morphogenesis were correlated with SPARCL1 (Fig. 9b). Their detailed outcomes of GSEA are displayed in Table 3. Glucose catabolic process and antigen procession and presentation were correlated with SPP1 (Fig. 9c), while mitotic sister chromatid segregation and mitotic nuclear division were associated with CHRDL1 (Fig. 9d). And their detailed results of GSEA are demonstrated in Additional file 10: Table S8.

\section{Immunohistochemical verification}

To increase the reliability of our findings, we investigated the distribution and expression of CHRDL1 and SPARCL1 proteins in five pairs of randomly selected tissues. Representative IHC images revealed that CHRDL1 and SPARCL1 proteins were primarily

(See figure on next page.)

Fig. 7 Prognostic roles of 10 hub genes and relation with pathological stages in patients of TCGA-LUAD dataset. Survival analysis for a CHRDL1, SPARCL1, SPP1, PENK, CYR61, CP, GAS6, GPC3, IL6 and WFS1 in LUAD. The LUAD patients are divided into high expression cohort (red) and low expression cohort (blue) according to the median expression of hub genes. Log-rank $\mathrm{P}<0.05$ is believed a statistical difference. $\mathbf{b}$ The relations of $\mathbf{b}$ CHRDL1, c SPARCL1, d SPP1 and e PENK with pathological stages among patients from TCGA-LUAD dataset 


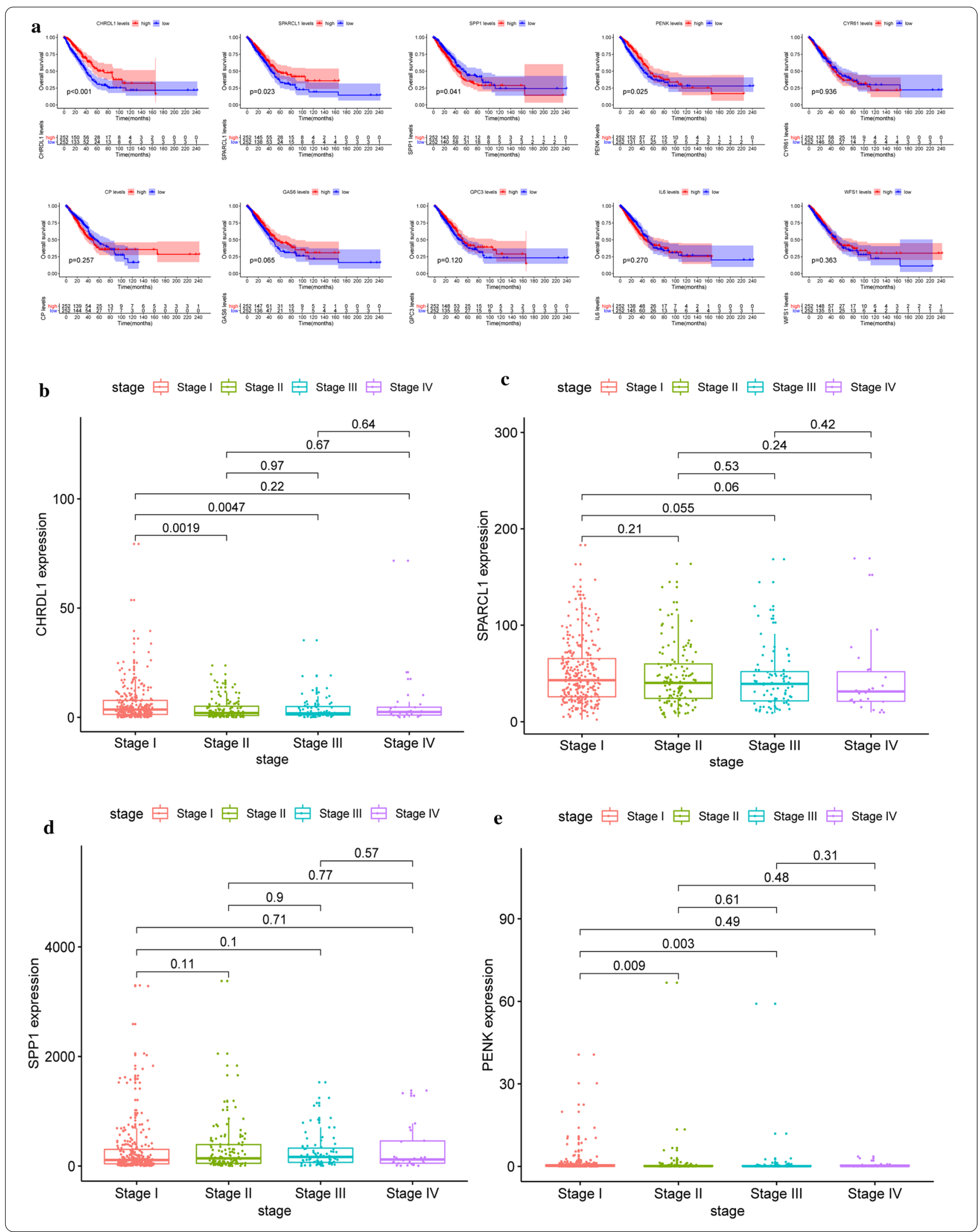


Table 1 Characteristics of the included datasets from GEO

\begin{tabular}{|c|c|c|c|c|c|c|}
\hline GEO datasets & Publication year & Country & RNA-Seq platforms & Normal & LUAD & Sum \\
\hline GSE10072 & 2008 & USA & GPL96 & 49 & 58 & 107 \\
\hline GSE116959 & 2019 & France & GPL17077 & 11 & 57 & 68 \\
\hline GSE19188 & 2010 & Netherlands & GPL570 & 65 & 45 & 110 \\
\hline GSE30219 & 2013 & France & GPL570 & 14 & 85 & 99 \\
\hline GSE31210 & 2011 & Japan & GPL570 & 20 & 226 & 246 \\
\hline GSE32863 & 2012 & USA & GPL6884 & 58 & 58 & 116 \\
\hline GSE33532 & 2014 & Germany & GPL570 & 20 & 40 & 60 \\
\hline GSE40791 & 2013 & USA & GPL570 & 100 & 94 & 194 \\
\hline GSE75037 & 2016 & USA & GPL6884 & 83 & 83 & 166 \\
\hline
\end{tabular}

GEO Gene Expression Omnibus, LUAD lung adenocarcinoma

distributed in the cytoplasm, partially on the cell membrane (Fig. 10a, b). Furthermore, both were obviously downregulated in LUAD tissues (Fig. 10c, d), which were consistent with our previous results.

\section{Discussion}

As a prevalent cancer associated with high mortality, lung cancer has resulted in substantial socioeconomic burdens to lung cancer patients and countries. Progress in LUAD therapy has been made in recent years, but the diagnosis and prognosis of LUAD remain poor because of the lack of precise molecular biomarkers. Thus, better indicators for the specific prognosis and progression of patients with LUAD are urgently required. In our analysis, a list of 486 differentially coexpressed genes was selected using the GSE75037 and TCGA-LUAD datasets through comprehensive bioinformatics analysis. These genes were significantly enriched in the regulation of epithelial cell proliferation, collagen-containing extracellular matrix, transforming growth factor beta binding and signaling pathways regulating pluripotency of stem cells. According to the rank of MCC scores, the top ten genes were identified as hub genes related to LUAD. Then, we found that 4 hub genes (namely, CHRDL1, SPARCL1, PENK and SPP1) of the top 10 genes were closely correlated with OS among patients with LUAD, and CHRDL1, SPARCL1 and PENK were positively correlated with the survival of LUAD, while SPP1 was negatively correlated with the prognosis. Based on external validation of the
GEO, CPTAC, and THPA databases, we observed that the mRNA and protein expression levels of CHRDL1, SPARCL1 and PENK were lower in LUAD, while SPP1 was upregulated. GSEA showed that DNA-dependent DNA replication and catalytic activity acting on RNA were associated with the expression of CHRDL1 and SPARCL1, respectively. Finally, the IHC outcomes validated the expression status of CHRDL1 and SPARCL1 in LUAD.

CHRDL1, namely, Chordin Like 1, is a specific antagonist of bone morphogenetic protein (BMP), and BMP signaling participates in many responses, including cell proliferation, migration and invasion in various cancers [20]. CHRDL1 was observed to be notably downregulated in many cancers [21]. Pei et al. observed that the CHRDL1 promoter was hypermethylated in gastric cancer, which may explain the downregulation of CHRDL1 in gastric cancer. Additionally, low expression of CHRDL1 was associated with worse survival among 100 patients with gastric cancer. In addition, these authors reported that the knockdown of CHRDL1 induced cell proliferation and metastasis via the activation of Akt and Erk, suggesting that CHRDL1 plays a tumor suppressor role in gastric cancer [22]. Wang et al. suggested that miRNA hsa-mir-204 contributed to cell proliferation, migration and invasion through the downregulation of CHRDL1 in gastric cancer [23]. Moreover, CHRDL1 could inhibit cell migration and invasion by suppressing BMP signaling in breast cancer [24]. CHRDL1 was found to be less

\footnotetext{
(See figure on next page.)

Fig. 8 External validation of the expression patterns of survival-related hub genes based on GSE19188 and Clinical Proteomic Tumor Analysis Consortium (CPTAC) database. The mRNA (a) and protein (b) expression patterns of CHRDL1 are compared between LUAD and normal lung tissues. The mRNA (c) and protein $(\mathbf{d})$ expression patterns of SPARCL1 are compared between LUAD and normal lung tissues. The mRNA (e) and protein (f) expression patterns of SPP1 are compared between LUAD and normal lung tissues. The mRNA (e) expression pattern of PENK is compared between LUAD and normal lung tissues
} 


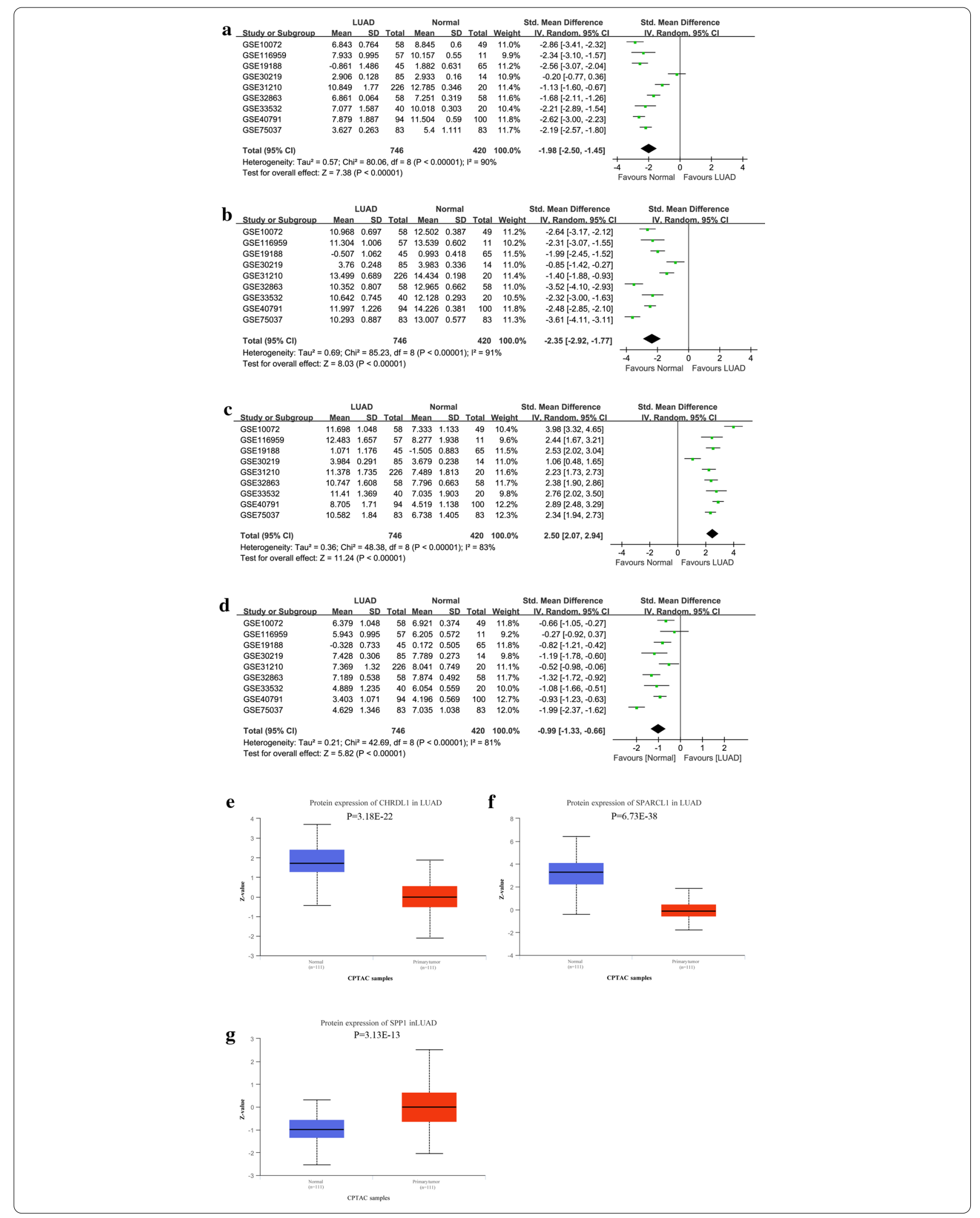




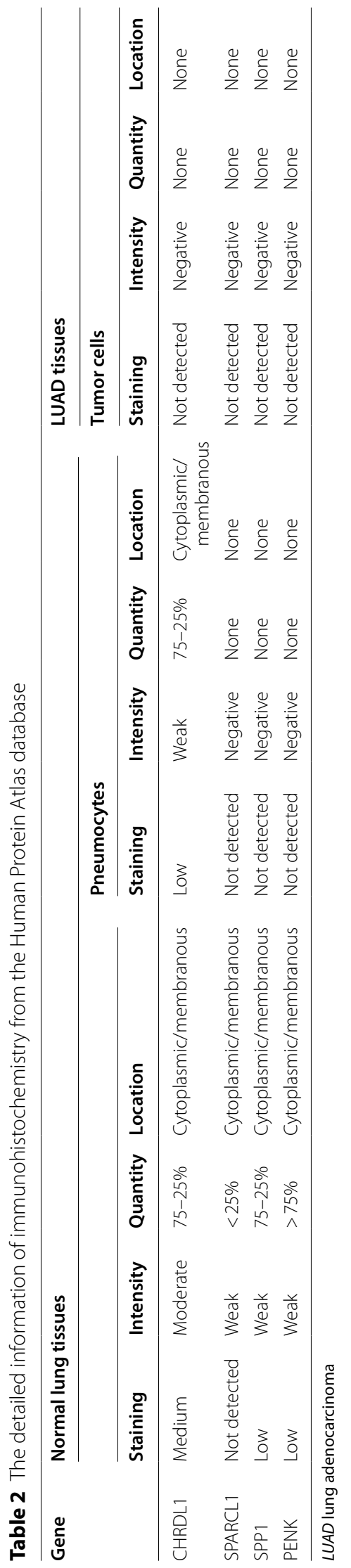




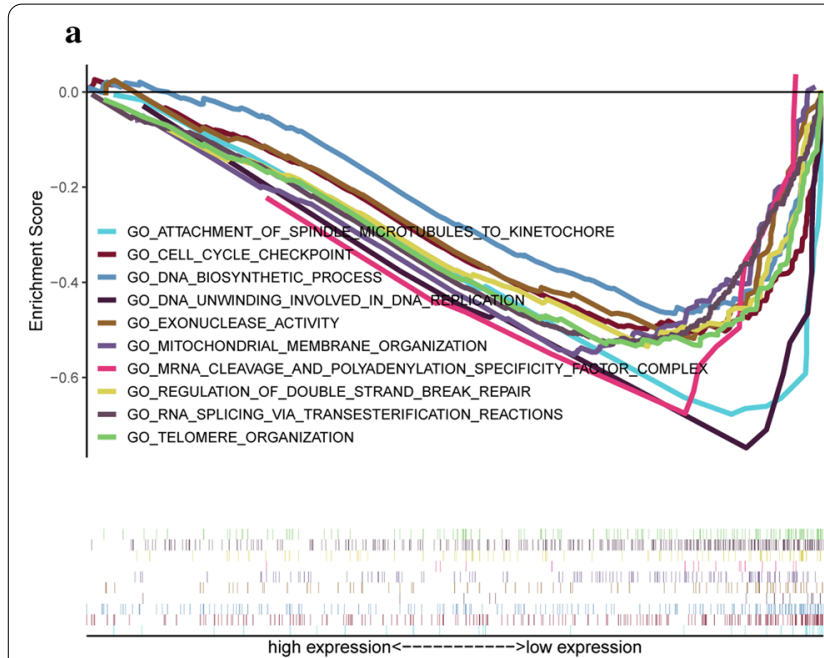

GSEA analysis of CHRDL1

c
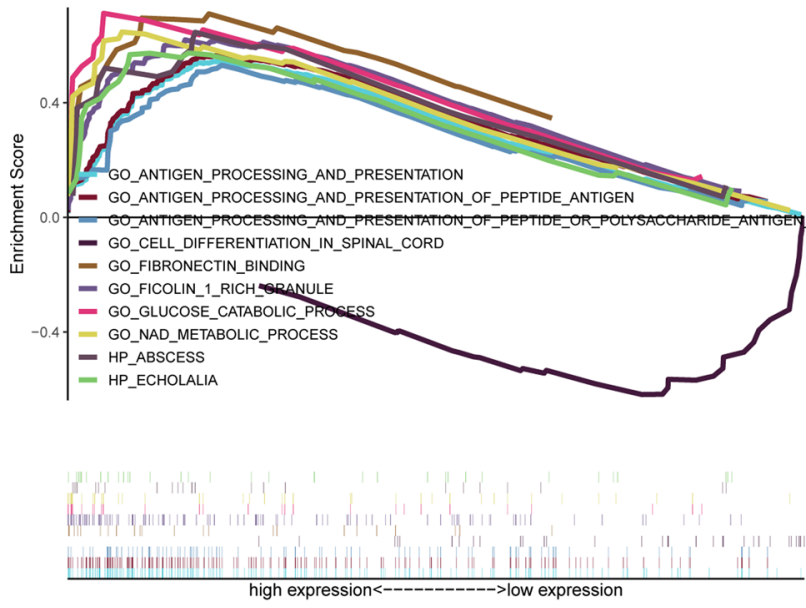

GSEA analysis of SPP1
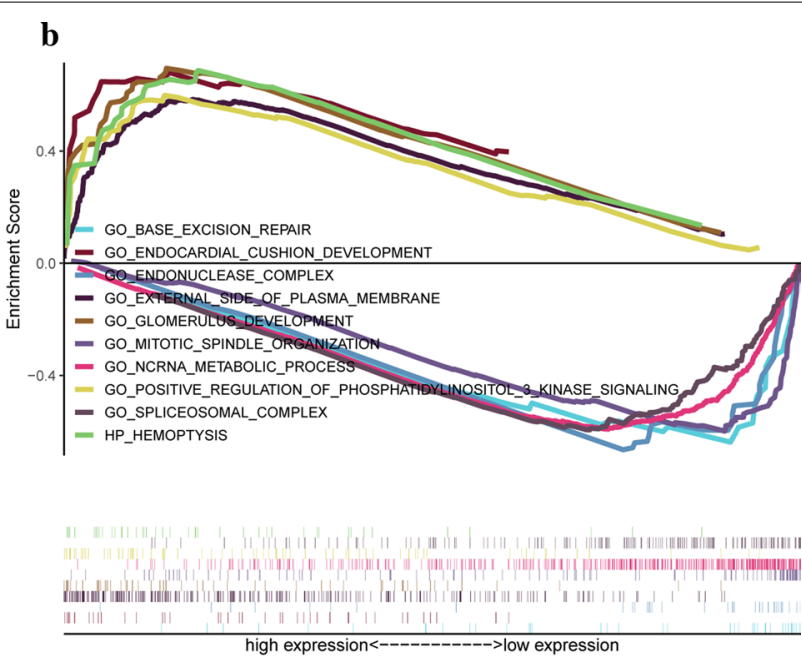

GSEA analysis of SPARCL1

\section{d}
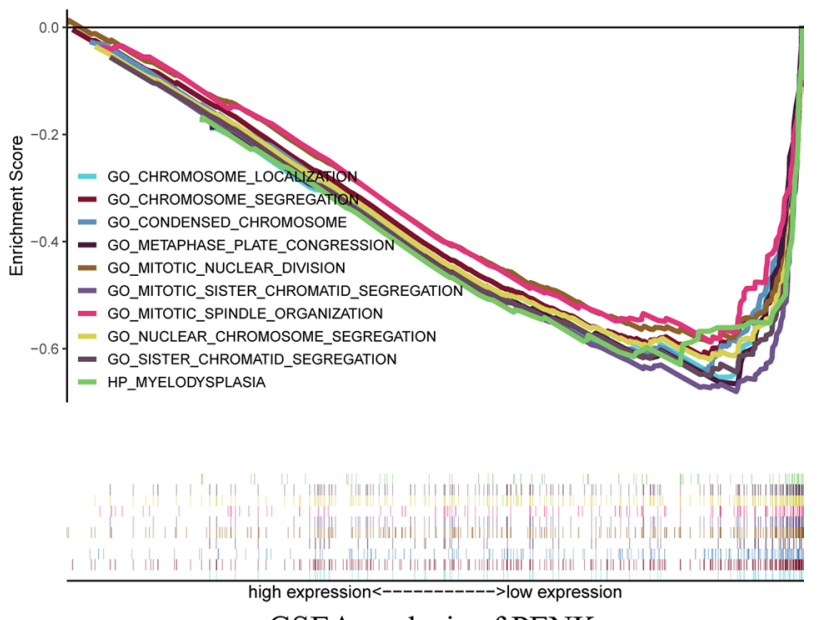

GSEA analysis of PENK

Fig. 9 Enrichment plots by gene set enrichment analysis (GSEA). Relative pathways associated with the expression of CHRDL1 (a), SPARCL1 (b), SPP1 (c), and PENK (d) are illustrated

expressed in thyroid cancer using the IHC method, and CHRDL1 was closely correlated with disease-free survival (DFS) of patients with thyroid cancer [25]. However, the role of CHRDL1 in LUAD has not been reported before our study, so additional studies exploring the role of CHRDL1 in LUAD are needed to further confirm our findings.

The secreted protein acidic rich in cysteine-like 1 (SPARCL1) is a matricellular protein that belongs to the SPARC-related protein family. SPARCL1 inhibited the progression of tumor cells from the G1 phase to the $S$ phase and participated in the negative regulation of cell proliferation [26]. Many studies have revealed the downregulated status and role of SPARCL1 in various cancers [27]. For example, SPARCL1 inhibited cell proliferation and invasion by inhibiting the mitogen-activated protein kinase kinase (MEK) and extracellular signal-related kinase (ERK) pathways in ovarian cancer [28]. Moreover, miR-539-3p was found to promote cell invasion by targeting SPARCL1 in epithelial ovarian cancer [29]. 
Table 3 Relative pathways associated with the expression of CHRDL1 and SPARCL1 using GSEA

\begin{tabular}{|c|c|c|c|c|c|}
\hline Gene & Name & ES & NES & $\begin{array}{l}\text { NOM } \\
\text { p-value }\end{array}$ & $\begin{array}{l}\text { FDR } \\
\text { q-value }\end{array}$ \\
\hline \multirow[t]{10}{*}{ CHRDL1 } & GO_REGULATION_OF_DOUBLE_STRAND_BREAK_REPAIR & -0.55 & -1.93 & $<0.0001$ & 0.109 \\
\hline & GO_CELL_CYCLE_CHECKPOINT & -0.51 & -1.93 & 0.006 & 0.107 \\
\hline & GO_DNA_UNWINDING_INVOLVED_IN_DNA_REPLICATION & -0.81 & -1.93 & $<0.0001$ & 0.106 \\
\hline & GO_EXONUCLEASE_ACTIVITY & -0.53 & -1.92 & 0.002 & 0.116 \\
\hline & GO_RNA_SPLICING_VIA_TRANSESTERIFICATION_REACTIONS & -0.53 & -1.92 & 0.006 & 0.114 \\
\hline & GO_TELOMERE_ORGANIZATION & -0.54 & -1.91 & 0.002 & 0.114 \\
\hline & GO_ATTACHMENT_OF_SPINDLE_MICROTUBULES_TO_KINETOCHORE & -0.71 & -1.91 & $<0.0001$ & 0.114 \\
\hline & GO_MITOCHONDRIAL_MEMBRANE_ORGANIZATION & -0.56 & -1.91 & 0.002 & 0.113 \\
\hline & GO_MRNA_CLEAVAGE_AND_POLYADENYLATION_SPECIFICITY_FACTOR_COMPLEX & -0.73 & -1.91 & 0.002 & 0.112 \\
\hline & GO_DNA_BIOSYNTHETIC_PROCESS & -0.47 & -1.91 & 0.002 & 0.112 \\
\hline \multirow[t]{10}{*}{ SPARCL1 } & GO_ENDONUCLEASE_COMPLEX & -0.68 & -2.17 & $<0.0001$ & 0.047 \\
\hline & GO_BASE_EXCISION_REPAIR & -0.67 & -2.11 & 0.002 & 0.048 \\
\hline & GO_SPLICEOSOMAL_COMPLEX & -0.6 & -2.1 & $<0.0001$ & 0.031 \\
\hline & GO_NCRNA_METABOLIC_PROCESS & -0.59 & -2.1 & $<0.0001$ & 0.048 \\
\hline & GO_MITOTIC_SPINDLE_ORGANIZATION & -0.61 & -2.09 & $<0.0001$ & 0.048 \\
\hline & GO_GLOMERULUS_DEVELOPMENT & 0.69 & 2.27 & $<0.0001$ & 0.046 \\
\hline & HP_HEMOPTYSIS & 0.69 & 2.17 & $<0.0001$ & 0.033 \\
\hline & GO_EXTERNAL_SIDE_OF_PLASMA_MEMBRANE & 0.58 & 2.16 & $<0.0001$ & 0.048 \\
\hline & GO_ENDOCARDIAL_CUSHION_DEVELOPMENT & 0.68 & 2.16 & $<0.0001$ & 0.047 \\
\hline & GO_POSITIVE_REGULATION_OF_PHOSPHATIDYLINOSITOL_3_KINASE_SIGNALING & 0.6 & 2.16 & $<0.0001$ & 0.047 \\
\hline
\end{tabular}

GSEA gene set enrichment analysis, NES normalized enrichment score, NOM nominal, FDR false discovery rate

SPARCL1 was downregulated in gastrointestinal stromal tumors, which contributed to cell migration and invasion, and SPARCL1 can predict the prognosis of gastrointestinal stromal tumors $(\mathrm{P}=0.008)$ [30]. Similarly, higher expression of SPARCL1 was correlated with better cell differentiation and less distant metastasis in colorectal cancers than those with lower expression of SPARCL1, and SPARCL1 was positively correlated with the prognosis of colorectal cancers $(\mathrm{P}<0.01)$ [31]. Wang et al. observed that SPARCL1 was a DNA methylation-regulated gene, and this gene was downregulated in LUAD [32]. Considering these reports and our findings, we can conclude that SPARCL1 and CHRDL1 play therapeutic and prognostic roles in the carcinogenesis and metastasis of LUAD.

Admittedly, several limitations existed in this analysis. (1) Integrated bioinformatics analysis was performed in our study to identify candidate prognostic genes in LUAD, but it might not be highly accurate for patients with each LUAD subtype. (2) Although the
GSE75037 and TCGA-LUAD datasets had many LUAD samples, only the two datasets were included in our analysis. (3) We did not validate our findings by conducting further experiments in addition to IHC, and more relevant basic experiments are required for further validation.

\section{Conclusion}

In general, this analysis was performed to find hub genes that might be correlated with the initiation and progression of LUAD using differential gene expression analysis and WGCNA. Ten hub genes were identified according to the rank of MCC values, and four genes were significantly associated with OS. Furthermore, CHRDL1 and SPARCL1 were candidate therapeutic and prognostic biomarkers of LUAD. 


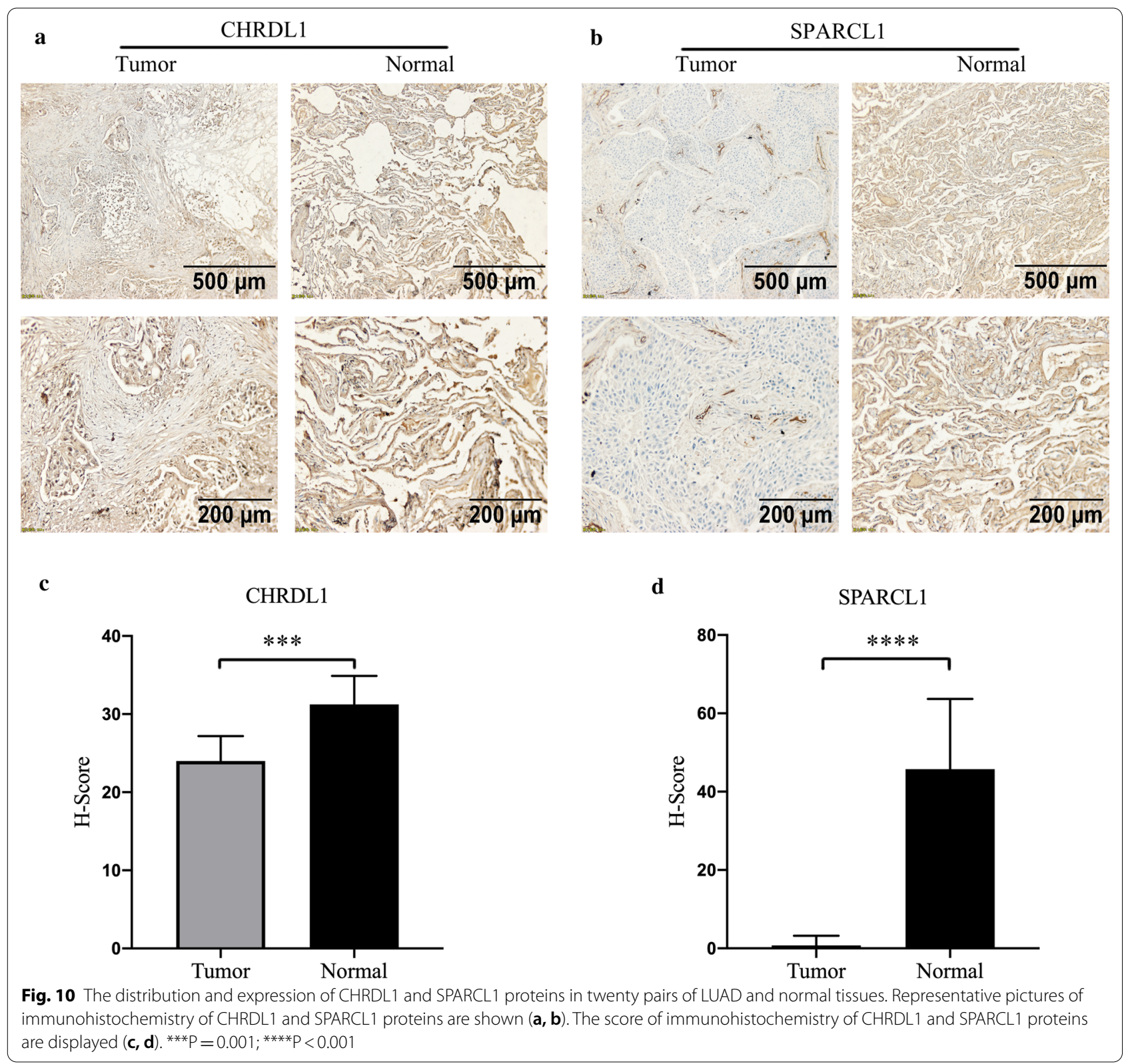

\section{Abbreviations}

NSCLC: Non-small cell lung cancer; LUAD: Lung adenocarcinoma; WGCNA: Weighed Gene Co-expression Network Analysis; DEGs: Differentially expressed genes; GEO: Gene Expression Omnibus; TCGA: The Cancer Genome Atlas; CPTAC: Clinical Proteomic Tumor Analysis Consortium; THPA: The Human Protein Atlas; FDR: False discovery rate; TOM: Topological overlap matrix; FPKM: Fragments per kilobase per million; TPM: Transcript per million; MCC: Maximal clique centrality; IHC: Immunohistochemistry; NES: Normalized enrichment score; GO: Gene ontology; KEGG: Kyoto encyclopedia of genes and genomes pathway; PPI: Protein-protein interaction network; STRING: Search Tool for the Retrieval of Interacting Genes; MCODE: Molecular Complex Detection; BP: Biological processes; CC: Cellular component; MF: Molecular function; OS: Overall survival; HR: Hazard ratio; GSEA: Gene set enrichment analysis; BMP: Bone morphogenetic protein; DFS: Disease-free survival; MEK: Mitogen-activated protein kinase kinase; ERK: Extracellular signal-related kinase. 


\section{Supplementary Information}

The online version contains supplementary material available at https://doi. org/10.1186/s12935-021-01933-9.

Additional file 1: Figure S1. External validation of the expression levels of survival-related hub genes based on the Human Protein Atlas (THPA) database. The immunohistochemistry $(\mathrm{IHC})$ results of CHRDL1 (A) and SPARCL1 (B) are compared between LUAD and normal lung tissues.

Additional file 2: Figure S2. External validation of the expression levels of survival-related hub genes based on the Human Protein Atlas (THPA) database. SPP1 (A), and PENK (B) are compared between LUAD and normal lung tissues.

Additional file 3: Table S1. The search strategy and selection criteria.

Additional file 4: Table S2. Major demographic and clinicopathological characteristics of patients with LUAD.

Additional file 5: Table S3. The differentially expressed genes (DEGs) in GSE75037.

Additional file 6: Table S4. The differentially expressed genes (DEGs) in TCGA-LUAD dataset.

Additional file 7: Table S5. The co-expression genes in the blue module of GSE75037.

Additional file 8: Table S6. The co-expression genes in the blue module of TCGA-LUAD dataset.

Additional file 9: Table S7. The intersection of genes among the two lists of differentially expressed genes (DEGs) and the two lists of co-expression genes.

Additional file 10: Table S8. Relative pathways associated with the expression of SPP1 and PENK using GSEA.

\section{Acknowledgements}

The authors gratefully acknowledge contributions from the GEO, TCGA, and CPTAC databases.

\section{Authors' contributions}

$\mathrm{HD}$ and $\mathrm{QH}$ had full access to all of the data in the manuscript and take responsibility for the integrity of the data and the accuracy of the data analysis. Concept and design: all authors. Acquisition, analysis, and interpretation of data: all authors. Drafting of the manuscript: HD and QH. Critical revision of the manuscript for important intellectual content: all authors. Statistical analysis: $\mathrm{HD}$ and QH. Supervision: MC. All authors read and approved the final manuscript.

\section{Funding}

This study is supported by the National Natural Science Foundation of China (Grant No. 81672972), and Zhejiang Provincial Key Research and Development Program (Grant No. 2019C03003), without the involvement of commercial entities. The funder had no role in the design or performance of the study; the collection, management, analysis, and interpretation of the data; the preparation, review, and approval of the manuscript; or the decision to submit the manuscript for publication.

\section{Availability of data and materials}

The datasets downloaded and analyzed during the current study are available on the TCGA and GEO database: TCGA-LUAD database: https://portal.gdc. cancer.gov/; GSE75037: https://www.ncbi.nlm.nih.gov/geo/query/acc.cgi? acc=GSE75037; GSE19188: https://www.ncbi.nlm.nih.gov/geo/query/acc.cgi? $\mathrm{acc}=\mathrm{GSE} 19188$.

\section{Declarations}

Ethics approval and consent to participate

Not applicable.

\section{Consent for publication}

Not applicable.

\section{Competing interests}

The authors declare that they have no competing interests.

\section{Author details}

${ }^{1}$ College of Life Sciences, University of the Chinese Academy of Sciences, Beijing 100049, China. ${ }^{2}$ Department of Radiation Oncology, Cancer Hospital of the University of Chinese Academy of Sciences (Zhejiang Cancer Hospital), Hangzhou 310022, China. Institute of Cancer Research and Basic Medical (IBMC), Chinese Academy of Sciences, Hangzhou 310022, China. ${ }^{4}$ Department of Radiation Oncology, Zhejiang Key Laboratory of Radiation Oncology, Zhejiang Cancer Hospital, Hangzhou 310022, China. ${ }^{5}$ Zhejiang Chinese Medicinal University, Hangzhou 310022, China. ${ }^{6}$ Jiangxi Medical College, Nanchang University, Nanchang 331800, China.

Received: 15 March 2021 Accepted: 13 April 2021

Published online: 12 May 2021

\section{References}

1. Siegel RL, Miller KD, Fuchs HE, Jemal A. Cancer statistics, 2021. CA Cancer J Clin. 2021;71(1):7-33.

2. Remon J, Hendriks LE, Cabrera C, Reguart N, Besse B. Immunotherapy for oncogenic-driven advanced non-small cell lung cancers: Is the time ripe for a change? Cancer Treat Rev. 2018;71:47-58.

3. Fang C, Wang L, Gong C, Wu W, Yao C, Zhu S. Long non-coding RNAs: how to regulate the metastasis of non-small-cell lung cancer. J Cell Mol Med. 2020;24(6):3282-91.

4. Li A, Bergan RC. Clinical trial design: past, present, and future in the context of big data and precision medicine. Cancer. 2020;126(22):4838-46.

5. Arora I, Tollefsbol TO. Computational methods and next-generation sequencing approaches to analyze epigenetics data: profiling of methods and applications. Methods. 2021;187:92-103.

6. Zhou J, Guo H, Liu L, Hao S, Guo Z, Zhang F, et al. Construction of coexpression modules related to survival by WGCNA and identification of potential prognostic biomarkers in glioblastoma. J Cell Mol Med. 2021;25(3):1633-44.

7. Zhou Q, Zhou LQ, Li SH, Yuan YW, Liu L, Wang JL, et al. Identification of subtype-specific genes signature by WGCNA for prognostic prediction in diffuse type gastric cancer. Aging. 2020;12(17):17418-35.

8. Reddy RRS, Ramanujam MV. High throughput sequencing-based approaches for gene expression analysis. Methods Mol Biol. 2018:1783:299-323.

9. Robinson MD, McCarthy DJ, Smyth GK. edgeR: a Bioconductor package for differential expression analysis of digital gene expression data. Bioinformatics. 2010;26(1):139-40.

10. Law CW, Alhamdoosh M, Su S, Dong X, Tian L, Smyth GK, et al. RNA-seq analysis is easy as 1-2-3 with limma, Glimma and edgeR. F1000Res. 2016. https://doi.org/10.12688/f1000research.9005.3.

11. Yu G, Wang LG, Han Y, He QY. clusterProfiler: an R package for comparing biological themes among gene clusters. OMICS. 2012;16(5):284-7.

12. Kanehisa M, Furumichi M, Tanabe M, Sato Y, Morishima K. KEGG: new perspectives on genomes, pathways, diseases and drugs. Nucleic Acids Res. 2017:45(D1):D353-d361.

13. Franceschini A, Szklarczyk D, Frankild S, Kuhn M, Simonovic M, Roth A, et al. STRING v9.1: protein-protein interaction networks, with increased coverage and integration. Nucleic Acids Res. 2013;41(Database issue):D808-15.

14. Smoot ME, Ono K, Ruscheinski J, Wang PL, Ideker T. Cytoscape 2.8: new features for data integration and network visualization. Bioinformatics. 2011;27(3):431-2.

15. Bandettini WP, Kellman P, Mancini C, Booker OJ, Vasu S, Leung SW, et al. Multicontrast delayed enhancement (MCODE) improves detection of subendocardial myocardial infarction by late gadolinium enhancement cardiovascular magnetic resonance: a clinical validation study. J Cardiovasc Magn Reson. 2012;14(1):83. 
16. Chin $\mathrm{CH}$, Chen $\mathrm{SH}$, Wu HH, Ho CW, Ko MT, Lin CY. cytoHubba: identifying hub objects and sub-networks from complex interactome. BMC Syst Biol. 2014;8(Suppl 4):S11.

17. Thul PJ, Lindskog C. The human protein atlas: a spatial map of the human proteome. Protein Sci. 2018;27(1):233-44.

18. Wu P, Heins ZJ, Muller JT, Katsnelson L, de Bruijn I, Abeshouse AA, et al. Integration and analysis of CPTAC proteomics data in the context of cancer genomics in the cBioPortal. Mol Cell Proteom. 2019;18(9):1893-8.

19. Liberzon A, Subramanian A, Pinchback R, Thorvaldsdóttir H, Tamayo P, Mesirov JP. Molecular signatures database (MSigDB) 3.0. Bioinformatics. 2011:27(12):1739-40.

20. Ren J, Wang Y, Ware T, laria J, Ten Dijke P, Zhu HJ. Reactivation of BMP signaling by suboptimal concentrations of MEK inhibitor and FK506 reduces organ-specific breast cancer metastasis. Cancer Lett. 2020;493:41-54.

21. Wang X, Gao C, Feng F, Zhuang J, Liu L, Li H, et al. Construction and analysis of competing endogenous RNA networks for breast cancer based on TCGA dataset. Biomed Res Int. 2020;2020:4078596.

22. Pei YF, Zhang YJ, Lei Y, Wu WD, Ma TH, Liu XQ. Hypermethylation of the CHRDL1 promoter induces proliferation and metastasis by activating Akt and Erk in gastric cancer. Oncotarget. 2017;8(14):23155-66.

23. Wang J, Ding $Y$, Wu $Y$, Wang $X$. Identification of the complex regulatory relationships related to gastric cancer from IncRNA-miRNA-mRNA network. J Cell Biochem. 2020;121(1):876-87.

24. Cyr-Depauw C, Northey JJ, Tabariès S, Annis MG, Dong Z, Cory S, et al. Chordin-like 1 suppresses bone morphogenetic protein 4-induced breast cancer cell migration and invasion. Mol Cell Biol. 2016;36(10):1509-25.

25. Shen Y, Dong S, Liu J, Zhang L, Zhang J, Zhou H, et al. Identification of potential biomarkers for thyroid cancer using bioinformatics strategy: a study based on GEO datasets. Biomed Res Int. 2020;2020:9710421.
26. Gagliardi F, Narayanan A, Mortini P. SPARCL1 a novel player in cancer biology. Crit Rev Oncol Hematol. 2017;109:63-8.

27. Gagliardi F, Narayanan A, Gallotti AL, Pieri V, Mazzoleni S, Cominelli M, et al. Enhanced SPARCL1 expression in cancer stem cells improves preclinical modeling of glioblastoma by promoting both tumor infiltration and angiogenesis. Neurobiol Dis. 2020;134:104705.

28. Ma Y, XUY, Li L. SPARCL1 suppresses the proliferation and migration of human ovarian cancer cells via the MEK/ERK signaling. Exp Ther Med. 2018;16(4):3195-201.

29. Gong YB, Fan XH. MiR-539-3p promotes the progression of epithelial ovarian cancer by targeting SPARCL1. Eur Rev Med Pharmacol Sci. 2019;23(6):2366-73.

30. Shen C, Yin Y, Chen H, Wang R, Yin X, Cai Z, et al. Secreted protein acidic and rich in cysteine-like 1 suppresses metastasis in gastric stromal tumors. BMC Gastroenterol. 2018;18(1):105.

31. Hu H, Zhang H, Ge W, Liu X, Loera S, Chu P, et al. Secreted protein acidic and rich in cysteines-like 1 suppresses aggressiveness and predicts better survival in colorectal cancers. Clin Cancer Res. 2012:18(19):5438-48.

32. Wang J, Yu XF, Ouyang N, Zhao SY, Guan XF, Yao HP, et al. Expression and prognosis effect of methylation-regulated SLIT3 and SPARCL1 genes in smoking-related lung adenocarcinoma. Zhonghua Yi Xue Za Zhi. 2019;99(20):1553-7.

\section{Publisher's Note}

Springer Nature remains neutral with regard to jurisdictional claims in published maps and institutional affiliations.
Ready to submit your research? Choose BMC and benefit from:

- fast, convenient online submission

- thorough peer review by experienced researchers in your field

- rapid publication on acceptance

- support for research data, including large and complex data types

- gold Open Access which fosters wider collaboration and increased citations

- maximum visibility for your research: over $100 \mathrm{M}$ website views per year

At BMC, research is always in progress.

Learn more biomedcentral.com/submissions 